\title{
A Separable Two-Dimensional Discrete Hartley Transform
}

Andrew B. Watson and Allen Poirson

December 1985
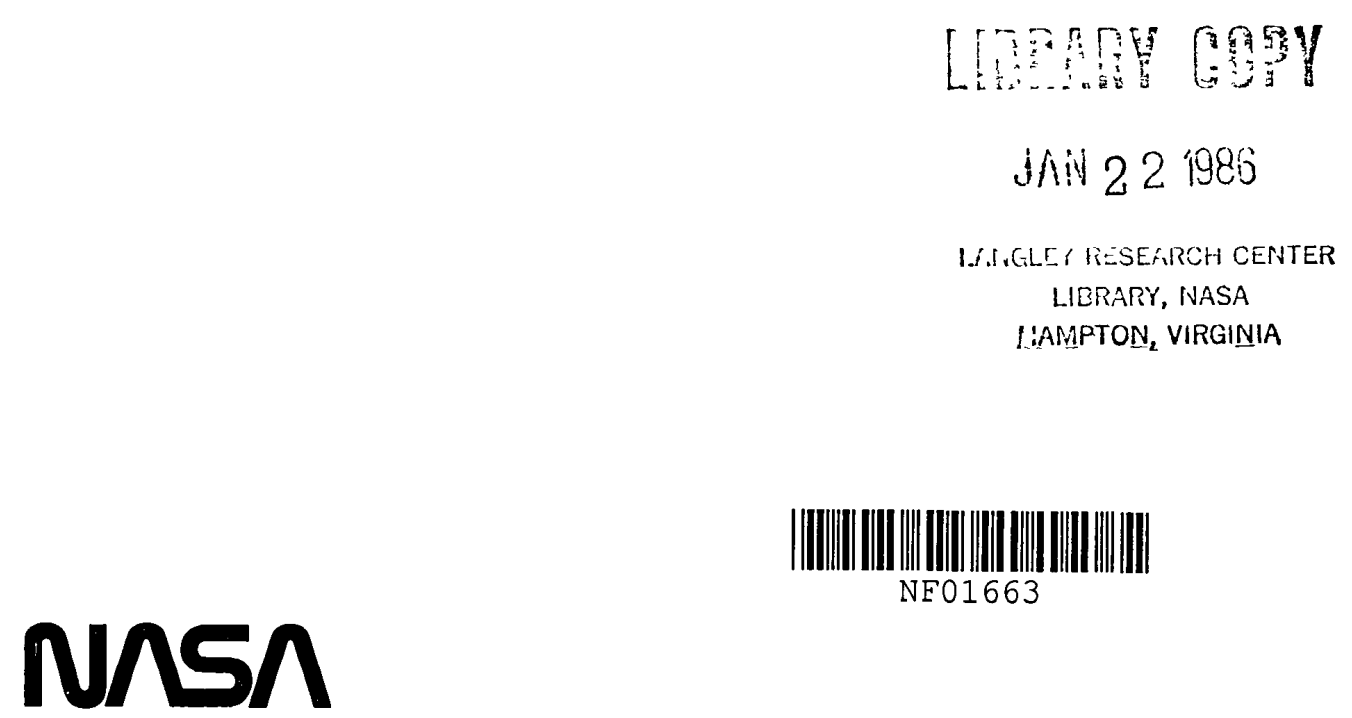

NF01663

Natıonal Aeronautıcs and

Space Admınıstratıon 


\section{A Separable Two-Dimensional Discrete Hartley Transform}

Andrew B. Watson, Ames Research Center, Moffett Field, California Allen Poirson, Stanford University, Palo Alto, California

December 1985

\section{N/Sก}

Natıonal Aeronautics and

Space Admınıstratıon

Ames Research Center

Moffett Field, Calıfornia 94035

$$
N 86-16907^{\#}
$$




\title{
A SEPARABLE TWO-DIMENSIONAL DISCRETE HARTLEY TRANSFORM
}

\author{
Andrew B. Watson and Allen Poirson \\ Perception and Cognition Group, NASA Ames Research Center \\ Moffett Field, CA 94035
}

Abstract-Bracewell has proposed the Discrete Hartley Transform (DHT) as a substitute for the Discrete Fourier Transform (DFT), particularly as a means of convolution (R. N. Bracewell, "Discrete Hartley transform," Journal of the Optical Society of America, vol. 73 , pp. 1832-1835, 1983 ). Here we show that the most natural extension of the DHT to two dimensions fails to be separable in the two dimensions, and is therefore inefficient. We consider an alternative separable form, and derive a corresponding convolution theorem. We also argue that the DHT is unlikely to provide faster convolution than the DFT. 


\section{Introduction}

In a series of recent papers Bracewell has drawn attention to the Hartley transform as a substitute for the much more widely known Fourier transform. $[1,2,3] \mathrm{He}$ has introduced a discrete Hartley transform (DHT) as an analog to the discrete Fourier transform (DFT), and has developed a fast algorithm (FHT) to compete with the Fast Fourier Transform (FFT). The purpose of this paper is to point out certain problems encountered in the extension of the DHT from one dimension (1D) to two dimensions (2D). There are two possible ways in which this extension can be made. Each has its virtues, but only one is practical. This choice requires a new convolution theorem, which we provide. However, even with this theorem, the 2D DHT appears not to provide a computational advantage over the DFT.

\section{The Discrete Fourier Transform (DFT)}

Consider a discrete sequence $p(x), x=0, \cdots, N-1$. The forward DFT of $p(x)$ is given by

$$
P_{F}(u)=\sum_{x=0}^{N-1} p(x) \exp (-i 2 \pi u x / N)
$$

and the inverse DFT,

$$
p(x)=\frac{1}{N} \sum_{u=0}^{N-1} P_{F}(u) \exp (i 2 \pi u x / N)
$$

In our notation we depart slightly from Bracewell, who places the : salar $1 / N$ in the forward transform in both the DFT and the DHT. This differs from general practice, $[4,5,6]$ and is less efficient if forward transforms are more common than reverse transforms. We therefore place the scalar in the inverse transform in our definitions of 
the DFT, DHT, and SDHT (defined below).

\section{Symmetry in the DFT of a real sequence}

Although the DHT is defined for both real and complex sequences, its practical value arises from the way in which it takes advantage of the symmetry in the DFT of a real sequence. Therefore in what follows we consider only real input sequences. A real sequence, defined by $N$ real numbers, has a DFT that contains $N$ complex entries, or $2 N$ real numbers. However, only $N$ of the $2 N$ real transform values are independent because the transform is Hermitian, that is, its real part is even and its imaginary part is odd. The Hartley transform is one way of taking advantage of this redundancy. Note that the sum (or difference) of an odd and an even sequence can be easily broken apart again into its odd and even parts. Thus because real and imaginary parts of the DFT are even and odd respectively, we can add them together with the assurance that they can be broken apart to recover the full complex DFT when desired. We therefore define the 1D DHT of a real sequence $p(x)$ as the real part minus the imaginary part of the DFT,

$$
P_{H}(u)=\operatorname{Re} P_{F}(u)-\operatorname{Im} P_{F}(u)
$$

Expanding the right side by means of Eq. 1,

$$
\begin{gathered}
P_{H}(u)=\sum_{x=0}^{N-1} p(x)[\cos 2 \pi u x / N+\sin 2 \pi u x / N] \\
P_{H}(u)=\sum_{x=0}^{N-1} p(x) \cos (2 \pi u x / N)
\end{gathered}
$$

The sequence cas defined by

$$
\operatorname{cas}(2 \pi u x / N)=\cos (2 \pi u x / N)+\sin (2 \pi u x / N)
$$

therefore serves as the basis sequence for the DHT. Note that the DHT may be 
expressed as the sum of its even and odd parts

$$
P_{H}(u)=P_{H E}(u)+P_{H O}(u)
$$

where

$$
P_{H E}(u)=\frac{1}{2}\left[P_{H}(u)+P_{H}(-u)\right] \quad P_{H O}(u)=\frac{1}{2}\left[P_{H}(u)-P_{H}(-u)\right]
$$

Throughout the text we interpret all sequence arguments modulo the length of the sequence, so for example if $P(u)$ is a sequence of length $N$ then $P(-1)=P(N-1)$.

Note that the DFT is easily reconstituted from the even and odd parts of the DHT

$$
P_{F}(u)=P_{H E}(u)-i P_{H O}(u)
$$

The inverse transform is

$$
p(x)=\frac{1}{N} \sum_{u=0}^{N-1} P_{H}(u) \operatorname{cas}(2 \pi u x / N)
$$

The principal advantage of the DHT over the DFT, as pointed out by Bracewell, is that it transforms a real input into a real output. Furthermore, the convolution theorem can be expressed in terms of the DHT. While this expression involves four sequence products, rather than the single product of the DFT convolution theorem, the former are products of real quantities and will therefore require on the order of $1 / 4$ the number of real operations. Furthermore, if either of the inputs to the convolution is even, as is quite common in filtering applications, the result reduces to a single product of real sequences, requiring on the order of $1 / 4$ as many operations as convolution via the DFT.

\section{The DHT in two dimensions}

One of the most frequent applications of the DFT is in image processing, where it is frequently used to implement convolution. For the DHT to be of use in image processing it must be extensible to two (and more) dimensions, and these extensions must carry 
with them a simple form of the convolution theorem. In one of his papers, Bracewell has offered a definition of the 2D DHT. [2] But this definition does not agree with the general 1D definition he proposes as embodied in Eq. (9), or with the convolution theorem he has offered (Ref. 2, p. 1834).

There are two natural candidates for the extension of the DHT to two dimensions. The basis functions of these two possibilities are

$$
\operatorname{cas}[2 \pi(u x / N+v y / M)] \quad \text { and } \quad \operatorname{cas}(2 \pi u x / N) \operatorname{cas}(2 \pi v y / M)
$$

In what follows, we will consider the advantages of each alternative.

First, which definition is consistent with the definition of the Hartley as the difference of even and odd parts of the DFT? Recall the observation that motivated the construction of the 1D DHT, namely that the DFT of a real input is Hermitian. Let $p(x, y)$ be a real 2D sequence (image) with $x=0, \ldots, N-1$ and $y=0, \ldots, M-1$. In two dimensions, odd-ness and even-ness are defined with respect to reflections about both axes:

$$
\begin{aligned}
& \text { Even }\{p(x, y)\}=p_{E}(x, y)=\frac{1}{2}[p(x, y)+p(-x,-y)] \\
& \text { Odd }\{p(x, y)\}=p_{O}(x, y)=\frac{1}{2}[p(x, y)-p(-x,-y)] .
\end{aligned}
$$

Under these definitions, the real part of the DFT of a real input is even, and the imaginary part is odd. We can again define the DHT to be the even part minus the odd part of the DFT,

$$
\begin{aligned}
P_{H}(u, v) & =\operatorname{Re} P_{F}(u, v)-\operatorname{Im} P_{F}(u, v) \\
& =P_{H E}(u, v)+P_{H O}(u, v) .
\end{aligned}
$$

In this case the DFT, as for $1 \mathrm{D}$, is given by 


$$
P_{F}(u, v)=P_{H E}(u, v)-i P_{H O}(u, v) .
$$

To discover the basis sequences of the 2D DHT we expand Eq. (15). Let $a=2 \pi u x / N$ and $b=2 \pi v y / M$. Then

$$
\begin{aligned}
P_{H}(u, v) & =\sum_{x=0}^{N-1} \sum_{y=0}^{M-1} p(x, y)[\cos (a+b)+\sin (a+b)] \\
& =\sum_{x=0}^{N-1} \sum_{y=0}^{M-1} p(x, y) \cos (a+b) .
\end{aligned}
$$

Thus we see that a definition of the 2D DHT that preserves the symmetries of the $1 \mathrm{D}$ transform leads to the first of the two possible basis functions introduced above.

This definition, while it forms a natural extension of the $1 \mathrm{D}$ transform, and leads to the convolution theorem offered by Bracewell, $[2]$ has a serious drawback. Unlike the $2 \mathrm{D}$ DFT', it is not separable in $u$ and $v$. Fast algorithms for the 2D DFT rely upon separability, and a straightforward extension of the FHT to 2D would also require separability.

\section{The Separable 2D Hartley Transform}

As a solution to this dilemma, we consider an alternative definition of the 2D DHT, which we will call the Separable Discrete Hartley Transform (SDHT), defined by

$$
P_{S}(u, v)=\sum_{x=0}^{N-1} \sum_{y=0}^{M-1} p(x, y) \operatorname{cas}(2 \pi u x / N) \operatorname{cas}(2 \pi v y / M)
$$

and the inverse transform

$$
p(x, y)=\frac{1}{N M} \sum_{u=0}^{N-1} \sum_{0=0}^{M-1} P_{S}(u, v) \cos (2 \pi u x / N) \operatorname{cas}(2 \pi v y / M) .
$$

This is essentially the 2D DHT as given by Bracewell (only the position of the scale factor is different). [2] Note that both transforms are separable in $x$ and $y$, and can be 
executed by first applying a 1D DHT to each row and then a ID DHT to each column (or vice versa). Since this transform no longer obeys Eq. (9), and does not lead to the convolution theorem derieved therefrom, we must discover the relationship between the SDHT and the DFT, and derive a convolution theorem for the SDHT.

\section{Relation between SDHT and DFT}

In the SDHT we have gained separability but have lost the simple relationships between the DHT and the DFT, namely, $D H T=\operatorname{Re} D F T-\operatorname{Im} D F T$ and $D F T=E v e n$ DHT $-i$ Odd $D H T$. However, as we show below, a similarly simple relation exists for the SDHT and the DFT. We begin by expanding the cas terms in the definition of the SDHT,

$$
\begin{gathered}
P_{S}(u, v)=\sum_{x=0}^{N-1} \sum_{y=0}^{M-1} p(x, y)(\cos a+\sin a)(\cos b+\sin b) \\
=\sum_{x=0}^{N-1} \sum_{y=0}^{M-1} p(x, y)(\cos a \cos b+\sin a \sin b+\cos a \sin b+\sin a \cos b) \\
=\sum_{x=0}^{N-1} \sum_{y=0}^{M-1} p(x, y)[\cos (a-b)+\sin (a+b)] \\
=\sum_{x=0}^{N-1} \sum_{y=0}^{M-1} p(x, y)\{\operatorname{Re}[\cos (a-b)-i \sin (a-b)] \\
-\operatorname{Im}[\cos (a+b)-i \sin (a+b)]\} \\
=\operatorname{Re} P_{F}(u,-v)-\operatorname{Im} P_{F}(u, v)
\end{gathered}
$$

Thus the SDHT is equal to the real part of the DFT reflected about the $u$ axis, minus the imaginary part of the DFT. As might be imagined, it does not matter whether the reflection is about the $u$ or $v$ axis. 
Going in the other direction, we can expand the expression for the DFT,

$$
\begin{gathered}
P_{F}(u, v)=\operatorname{Re} P_{F}(u, v)+i \operatorname{Im} P_{F}(u, v) \\
=\frac{1}{2}\left\{\left[\operatorname{Re} P_{F}(u, v)+\operatorname{Re} P_{F}(-u,-v)-\operatorname{Im} P_{F}(u,-v)-\operatorname{Im} P_{F}(-u, v)\right]\right. \\
\left.-i\left[\operatorname{Re} P_{F}(-u, v)-\operatorname{Re} P_{F}(u,-v)+\operatorname{Im} P_{F}(u, v)-\operatorname{Im} P_{F}(-u,-v)\right]\right\} \\
=\frac{1}{2}\left[P_{S}(u,-v)+P_{S}(-u, v)\right]-\frac{i}{2}\left[P_{S}(u, v)+P_{S}(-u,-v) \text { bold }\right] \\
=P_{S E}(u,-v)-i P_{S O}(u, v) .
\end{gathered}
$$

\section{Summary of relationships}

For future reference we summarize the relationships among the DFT, DHT, and SDHT:

$$
\begin{gathered}
P_{S}(u, v)=P_{H O}(u, v)+P_{H E}(u,-v) \\
P_{S}(u, v)=\operatorname{Re} P_{F}(u,-v)-\operatorname{Im} P_{F}(u, v) \\
P_{H}(u, v)=P_{S O}(u, v)+P_{S E}(u,-v) \\
P_{H}(u, v)=\operatorname{Re} P_{F}(u, v)-\operatorname{Im} P_{F}(u, v) \\
P_{F}(u, v)=P_{S E}(u,-v)-i P_{S O}(u, v) \\
P_{F}(u, v)=P_{H E}(u, v)-i P_{H O}(u, v)
\end{gathered}
$$

\section{SDHT Convolution Theorem}

To be useful, the SDHT must yield a simple convolution theorem. We can derive this theorem from the preceding relations between DFT and SDHT. Let $r(x, y)$ be the result of convolving the two real sequences $p(x, y)$ and $q(x, y)$,

$$
r(x, y)=p(x, y) * q(x, y)=\sum_{i=0}^{N-1} \sum_{j=0}^{M-1} p(i, j) q(x-i, y-j) .
$$

From the convolution theorem for the DFT we have 


$$
R_{F}(u, v)=P_{F}(u, v) Q_{F}(u, v) .
$$

From Eq. (31) we can convert this to an expression for the SDHT,

$$
\begin{gathered}
R_{S}(u, v)=\operatorname{Re} R_{F}(u,-v)-\operatorname{Im} R_{F}(u, v) \\
=\operatorname{Re}\left[P_{F}(u,-v) Q_{F}(u,-v)\right]-\operatorname{Im}\left[P_{F}(u, v) Q_{F}(u, v)\right] .
\end{gathered}
$$

The next step is to substitute Eq. (34) for each DFT,

$$
\begin{aligned}
& =\operatorname{Re}\left\{\left[P_{S E}(u, v)-i P_{S O}(i,-v)\right]\left[Q_{S E}(u, v)-i Q_{S O}(u,-v)\right]\right\} \\
& -\operatorname{Im}\left\{\left[P_{S E}(u,-v)-i P_{S O}(u, v)\right]\left[Q_{S E}(u,-v)-i Q_{S O}(u, v)\right]\right\} .
\end{aligned}
$$

Thus we arrive at a convolution theorem

$$
\begin{gathered}
R_{S}(u, v)=P_{S E}(u, v) Q_{S E}(u, v)-P_{S O}(u,-v) Q_{S O}(u,-v) \\
\quad+P_{S E}(u,-v) Q_{S O}(u, v)+P_{S O}(u, v) Q_{S E}(u,-v) .
\end{gathered}
$$

\section{Symmetrical inputs}

Bracewell notes that if one of the inputs to a convolution is even, then the DHT of the convolution reduces to the product of the DHTs. For the SDHT, examination of Eq. (41) shows that if $p(x, y)$ is even, the SDHT of the convolution reduces to

$$
\begin{gathered}
R_{S}(u, v)=P_{S E}(u, v) Q_{S E}(u, v) \\
+P_{S O}(u,-v) Q_{S O}(u,-v)+P_{S E}(u,-v) Q_{S O}(u, v) .
\end{gathered}
$$

Thus only three products need to be computed.

\section{Complexity}

As noted above, the SDHT is one method of exploiting the symmetry of the DFT of a real sequence. Other well-known methods allow one to compute the DFT of a real sequence in essentially half the number of operations required for a complex sequence. [5] Let us call this sort of transform an RDFT. Furthermore, the result of an RDFT on a real sequence of length $\mathrm{N}$ is $N / 2$ complex values. Following Bracewell, let us assume 
for the moment that for a sequence of length $N$ the number of operations required by SDHT and RDFT are equal. The convolution via the RDFT will require $N / 2$ complex multiplies, or approximately $2 N$ real multiplies. Convolution via the SDHT will require $4 N$ real multiplies in the general case. When one of the inputs is symmetrical, then as noted above only $3 N$ real multiplies are required. In neither case, however, does the SDHT confer an advantage over the DFT.

\section{Summary}

We have shown that the most natural extension of the DHT to two dimensions fails to be separable in the two dimensions. We have examined an alternative, separable form for the 2D DHT, originally proposed by Bracewell. We have determined its relation to the DFT and have derived a corresponding convolution theorem. Finally, we note that the DHT is unlikely to provide faster convolution than the DHT in the general case.

\section{Acknowledgements}

We thank Albert J. Ahumada, Jr., Ronald N. Bracewell, and Brian A. Wandell for useful discussions. 


\section{References}

[1] R. V. L. Hartley, "A more symmetrical Fourier analysis applied to transmission problems," Proceedings of the Institute of Radio Engineers, vol. 30, pp. 144$150,1942$.

[2] R. N. Bracewell, "Discrete Hartley transform," Journal of the Optical Society of America, vol. 73, pp. 1832-1835, 1883.

[3] R. N. Bracewell, "The fast Hartley transform," Proceedings of the IEEE, vol. 72, pp. 1010-1018, 1984.

[4] H. J. Nussbaumer Fast Fourier transformation and convolution algorithms. New York: Springer-Verlag, 1982.

[5] IEEE Acoustics, Speech, and Signal Processing Committee Programs for digital signal processing. Ncw York: IEEE Press, 1979.

[6] A. V. Oppenheim and R. W. Schafer Digital signal processing. Englewood Cliffs, N.J.: Prentice Hall, 1975. 


\begin{tabular}{|c|c|c|c|c|}
\hline & $\begin{array}{l}\text { Report No. } \\
\text { NASA TM-88203 }\end{array}$ & 2 Government Accession No & \multicolumn{2}{|c|}{3 Recipient's Catalog No } \\
\hline & \multicolumn{2}{|l|}{ 4. Title and Subtitle } & \multicolumn{2}{|c|}{$\begin{array}{l}5 \text { Report Date } \\
\text { December } 1985\end{array}$} \\
\hline & \multicolumn{2}{|c|}{$\begin{array}{l}\text { A SEPARABLE TWO-DIMENSIONAL DISCRETE HARTLEY } \\
\text { TRANSFORM }\end{array}$} & \multicolumn{2}{|c|}{6 Performing Organization Code } \\
\hline 7 & \multicolumn{2}{|c|}{$\begin{array}{l}\text { Author(s) } \\
\text { Andrew B. Watson and Allen Poirson (Stanford } \\
\text { University, Palo Alto, CA) }\end{array}$} & \multicolumn{2}{|c|}{$\begin{array}{l}8 \text { Performing Organization Report No } \\
\text { A- } 86071\end{array}$} \\
\hline \multirow[t]{2}{*}{9} & \multicolumn{2}{|c|}{ Performing Organization Name and Address } & \multicolumn{2}{|c|}{10 Work Unit No } \\
\hline & \multicolumn{2}{|c|}{$\begin{array}{l}\text { Ames Research Center } \\
\text { Moffett Field, CA } 94035\end{array}$} & \multicolumn{2}{|c|}{ 11. Contract or Grant No. } \\
\hline & \multicolumn{2}{|l|}{ Sponsoring Agency Name and Address } & \multicolumn{2}{|c|}{$\begin{array}{l}13 \text { Type of Report and Period Covered } \\
\text { Technical Memorandum }\end{array}$} \\
\hline 12 & \multicolumn{2}{|c|}{$\begin{array}{l}\text { National Aeronautics and Space Administration } \\
\text { Washington, DC } 20546\end{array}$} & \multicolumn{2}{|c|}{14 Sponsoring Agency Code } \\
\hline 15 & \multicolumn{4}{|c|}{ 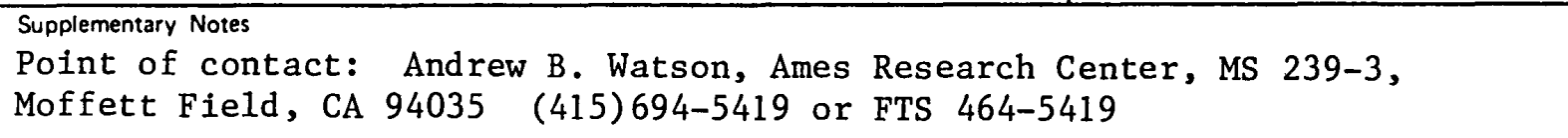 } \\
\hline 16 & \multicolumn{4}{|c|}{$\begin{array}{l}\text { Abstract } \\
\text { Bracewell has proposed the Discrete Hartley Transform (DHT) as a } \\
\text { substitute for the Discrete Fourier Transform (DFT), particularly as a } \\
\text { means of convolution (R. N. Bracewell, "Discrete Hartley transform," Journal } \\
\text { of the Optical Society of America, vol. } 73 \text {, pp. 1832-1835, 1983). Here we } \\
\text { show that the most natural extension of the DHT to two dimensions fails to } \\
\text { be separable in the two dimensions, and is therefore inefficient. We con- } \\
\text { sider an alternative separable form, and derive a corresponding convolution } \\
\text { theorem. We also argue that the DHT is unlikely to provide faster convolu- } \\
\text { tion than the DFT. }\end{array}$} \\
\hline 17 & $\begin{array}{l}\text { Key Words (Suggested by Author(s)) } \\
\text { Image processing } \\
\text { Convolution } \\
\text { Fourier trans form }\end{array}$ & $\begin{array}{l}18 \text { Distributic } \\
\text { Unlimi }\end{array}$ & 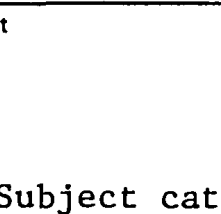 & ory -53 \\
\hline & $\begin{array}{l}\text { Security Classif (of this report) } \\
\text { Unclassified }\end{array}$ & $\begin{array}{l}20 \text { Security Classif (of this page) } \\
\text { Unclassified }\end{array}$ & $\begin{array}{c}21 \text { No of Pages } \\
14\end{array}$ & $\begin{array}{r}22 \text { Price }^{\circ} \\
\mathrm{A} 02\end{array}$ \\
\hline
\end{tabular}

-For sale by the National Technical Information Service, Springfield, Virginia 22161 
End of Document 